Canadian University Music Review

Revue de musique des universités canadiennes

\title{
In Memoriam Serge Garant
}

Gabriel Charpentier, André Prévost, R. Murray Schafer, Bruce Mather, Norma Beecroft, Robert Aitken, John Rea, Jean Papineau-Couture, José Evangelista, Anne Lauber, François Morel, Gilles Tremblay et Pierre Boulez

\section{Numéro 7, 1986}

URI : https://id.erudit.org/iderudit/1014083ar

DOI : https://doi.org/10.7202/1014083ar

Aller au sommaire du numéro

Éditeur(s)

Canadian University Music Society / Société de musique des universités canadiennes

ISSN

0710-0353 (imprimé)

2291-2436 (numérique)

Découvrir la revue

Citer ce document

Charpentier, G., Prévost, A., Schafer, R., Mather, B., Beecroft, N., Aitken, R., Rea, J., Papineau-Couture, J., Evangelista, J., Lauber, A., Morel, F., Tremblay, G. \& Boulez, P. (1986). In Memoriam Serge Garant. Canadian University Music Review / Revue de musique des universités canadiennes, (7), 4-25.

https://doi.org/10.7202/1014083ar

All Rights Reserved @ Canadian University Music Society / Société de musique des universités canadiennes, 1986
Ce document est protégé par la loi sur le droit d’auteur. L’utilisation des services d'Érudit (y compris la reproduction) est assujettie à sa politique d'utilisation que vous pouvez consulter en ligne.

https://apropos.erudit.org/fr/usagers/politique-dutilisation/ 


\section{SERGE GARANT, MORT}

Serge est le temps passé/présent de presque toute mon existence, celui auquel j'ai voulu toujours parler, à qui j'ai dit : "Ne crois-tu pas que .... C'est ça que tu voulais ? .... Il me semble que .... C'est peut-être ça .... C'EST A !" Et la page était tournée. Et on recommence ! On a recommencé, recommencé : des mots brefs, des regards entendus, à cet être intuitif, informé de toutes les limites, d'au-delà de toutes les limites, de toutes les respirations : à lui, la confession de tous les futurs.

Oui, j'ai forcé ses Offrandes ; puis, de cent mille mots offerts, il a su trouver la petite larme, le rire, et les éclats translucides de tous les gestes de la vie. Il reprenait toujours son chant secret, enrichi, multiplié, et si clair. L'âme.

Il aimait la musique et les musiciens, et les autres : il les découvrait à mesure, ou mesure par mesure. S'il crépitait parfois de la musique des autres, il savait ne jamais la détruire. Il était honnête et savant, et sans diplôme d'université, mais il avait une telle famille autour de lui à qui il apprenait à apprendre, à entendre, à déchiffrer les labyrinthes de toutes les créations, à comprendre, à se dépasser, puis, sans juger, il faisait sourdre le choix : il enseignait à aimer.

Serge, mort ? Si discret et fragile, mais précis et chaud, il vit de mémoire comme on fait la musique par coeur.

Gabriel Charpentier le 6 décembre 1986

Gabriel Charpentier nous invite à parcourir le labyrinthe qui suit, quatrième d'une série de constructions de même type entreprise par l'auteur depuis plus d'une décennie. Façonné en 1975, Labyrinth 4 convoque des événements artistiques auxquels Charpentier fut associé de près cette année-là, dont la composition et la production pour la télévision de ... chant d'amours, de Garant. 
\begin{tabular}{|l|l|l|l|l|l|l|}
\hline Labyrinth 4 & for PM & deciphering the illegible & revealing the invisible & defying the untransmittable & January 1975 & out \\
\hline
\end{tabular} \begin{tabular}{l|l|l|l|l|l|l|l|l}
\hline labyrinth Louis Riel Ottawa & out actors & out technicians & out public warmth lout risks and perils & the doors are flung wide, the
\end{tabular} \begin{tabular}{|l|l|l|l|l|l|l|l} 
machines have been forced & let's leave the stage & let's enter the picture & quickly living each second invading time & theatre \\
\hline
\end{tabular} time television time let's change time in labyrinth Serge Garant Chant d'Amours / creation: action of bringing into existence original production of human intelligence flashback: Rome, June 1974, Serge: "J'ai trouvé!". Montreal. November, work begins

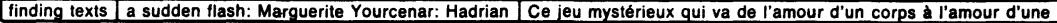
\begin{tabular}{|l|l|l|l|l|l|}
\hline personne... & to encircle love & to be, with, in, for Serge labyrinth of words, 1000 poems, 100000 words mingling of centuries \\
\hline
\end{tabular} \begin{tabular}{|l|l|l|l|l|l|}
\hline poets, lose names and faces, men and women in love & tears, cries and whispers & set me a seal upon thine heart & why does \\
\hline
\end{tabular} free love cost so much key word waves of words: sounds, rhythms, forms, colours / stage-fright lout labyrinth Garant | January 26 Louis Riel's doing fine, thank you L Les Grands Ballets Canadiens, Les Beaux Dimanches. Loves, Harry FreedmanBrian Macdonald re-creation: to give a new existence catching the present moment, the instant, the report, the event: \begin{tabular}{l|l|l|l|l|l|l|l}
\hline television time & on & that voice of Mary Morrison's & rush labyrinth Serge & delays delays delays & such is my love, to thee so \\
\hline
\end{tabular} I belong that for thy right myself will bear all wrong texts chosen at last /stage-fright If'll work out pictures lenter Pierre Morin, enter Hugo Wuetrich - at last labyrinth of architectural forms let's stop talking in thin air let's precariously settle in

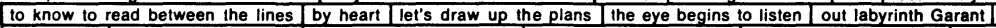
\begin{tabular}{|l|l|l|l|l|l|l|}
\hline February 4 & in labyrinth Gropus 7 & another team takes over & how io revitalize oneself & Garaudy: Danser sa vie & February 10 \\
\hline
\end{tabular} in labyrinth Jacques Offenbach in labyrinth La Belle Hélène theatre time, television time cut erase scratch out join up carve up and out, laugh the eye listens with thanks to Claudel the ears sees lout Hélène louch in Hélène / cut erase, etc etc, slash scalp behead quillotine, laugh laugh out Héléne in Héléne cut erase, etc etc, love vivisect resect laugh and cry recreation: action of being recreated by some pleasant occupation lout Hélène Lout Paul Buissonneau in Europe bon voyage March 6 in Jean Gascon TNM: Long Day's Journey into Night labyrinih of indelible loves in labyrinth Garant choosing :

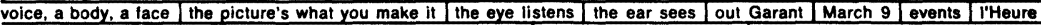
Espagnole, Les Beaux Dimanches $/$ theatre time, television time $/$ re-creation? recreation? 1 theatrical transparency of the music Saskatoon: Aomeo and Juliette, Les Grands Ballets Canadiens, Brian 1 Now old desire doth in his death bed lie and young affection gapes to be his heir in labyrinth Garant to feel sounds and pictures taking shake to feel the score spurt up to \begin{tabular}{l|l|l|l|l|l|l|l}
\hline feel the sels, emerge, boom high & March 18 in labyrinth Concerto & Mozart or Bach of Prokofiev or Mendelsohn or Bartok or \\
\hline
\end{tabular} Schumann or Saint-Saens 1 piano, violin, cella, etc. morning, midday, evening music? take your pick $/$ remembering new perceptions and discoveries all music is photogenic but. look out, you must know how [It's all a question of choice You'll be judged on your choice 1 April 2 Ce jeu mystérieux qui va de l'amour d'un corps a l'amour d'une personne m'a semblé assez

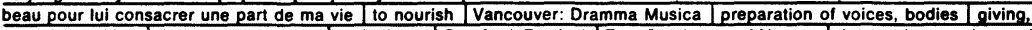
receiving, taking the next generation / revitalizing Strattord Festival Two Gentlemen of Verona theatre time - dry as dust April 14 at last, in studio, Concerto music is made with men, for men catching the maximum, with the camera as withess many-facetted eye of the producer and his watchful team I I call you: my friends, the cameras I my friends, the video-tape machines my friends, the microphones $/$ my friends, the tape recorders 1 all friendly, fragile, obedient, capricious, secret machines knowing how to use them in labyrinth Belle Hellène trimming polishing floating her off Bejart: "We violate, destroy, rebuild and give air to what we love. We make it live." in Concerto $/$ studio almost empty leaving room for the music the performers, the instruments / let all the counterpoints take form this tip of the soul takes shape /catching for fractions of seconds faces, breathing, secret, furtive, hidden gestures the alchemy of gilded, curving instruments in Hélène, la Belle Hélène, Hélène and again Hélène analyses, paper, paper, analyses, paper, scissors we're getting there, we'll finish, we're happy May 5 Héléne's finished recreation: see above if you can find it... in Concerto selling concertos! hanging together superimposing, mixing all styles, all pictures /madness indeed! Bach, Ravel, Prokofiev as bed-fellows It happens in the best \begin{tabular}{l|l|l|l|l} 
tamilies & revitalizing oneself & Bejart, Brussels & "So, what shall we do, together?", he said Paris, The Barber of Seville, Roncon
\end{tabular} version baroque, mad, incisive theatre oetting out the lace $/$ rediscovering Beaumarchais 1 May 28 in Chant d'Amours, in

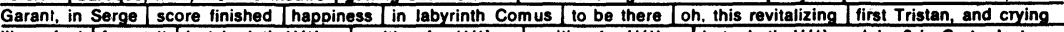

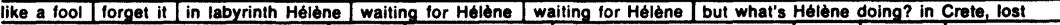

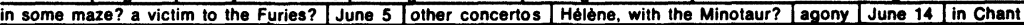
d'Amours Serge at Pierre's Pierre with Serge the number becomes sound 1 the sound becomes image for hours on end \begin{tabular}{|l|l|l|l|l|l}
\hline feeling the structure, multiple movement & rhythms great and small the song goes on singing for hours on end, a brightly lit
\end{tabular} round table Hélene, Héléne, in what limbo are you suffering? in Chant d'Amours first reading Let's unveil it majestically in the Kingdom Hall Let's be the first witnesses! Ensemble: collection of elements, in infinite number, likely to possess certain properties, and havirg certain relationships between each other, or with elements of other ensembles / strange accoucheurs armies of trumpets, harps, bells, voices, poems, papers, stop-watches it will be a fine baby Helène lost in the Underworld June 24 in Chant d'amours $/$ studio studio studio forgetting Hélène 1 magic cell $/$ white-hung womb brasses and chrome glitter and blues vibrate for three whole days ears eyes lears see 1 eyes hear men, women and machines Chant d'Amours is being born $/$ Chant d'Amours is being born at home 1 royal birth $/$ let's drink to it $/$ quick $/$ June 27 Heléne flayed \begin{tabular}{|l|l|l|l|l|l|l|l|l|l|l}
\hline alive & surgery & recreating: see above... & July 5 & in Dramma Musica & assuring, worrying, briefing the newcomers & revitalizing \\
\hline
\end{tabular} retrospective need for pictures / Mass for our time Set me as a seal upon thine heart 1 seeing angles, rhythms, faces, gestures physical need nourishing the future July 19 in Mozart Piano Concerto K 415 screeninglthinking, reflecting the music apology for the hand, the eye, the brain, the guts music comes right from the guts 1 mixture of techniques: Television? Film? reflecting the music, like a mirror $/$ so much combined sensitivity: composer, pertormers, producer, lechnicians... able to catch the eye and ear of that unknown public (Come with us. We've done our work. now do yours. We'll understand each

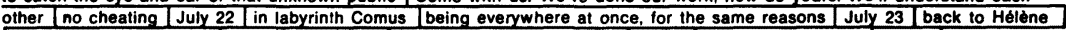

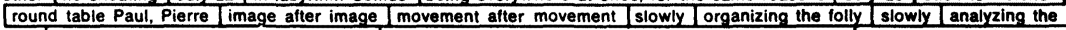
folly having one's feet firmly on the ground theatre time, laughing with a thousand others, together television time: hoping 10 make an unknown viewer laugh, behind his glass curtain / there are no set formulas / there are techniques / starting from \begin{tabular}{l|l|l|l|l|l|l|l|l|l|l}
\hline scratch & every time & July 26 & revitalizing oneself on for some sleep & Mount Orlord Mozart Ravel physical need to feel the \\
\hline
\end{tabular} \begin{tabular}{l|l|l|l|l|l|l|l|l}
\hline public & the warm-hearted unknown feeding oneself and botanizing & July 28 & in Helène llet's get away from the papers let's
\end{tabular} get to the heart of it let's pick up the shreds / slowly quick quick slowiy meticulously wonderful labyrinth lunbreathable suffocating these passionate buffoons will see the light /let's organize the light in-dept revitalization Les Grands Ballets Canadiens $\mid$ Béjart-Stravinsky: Oiseau de Feu 1 Bejart: "Choreography's made by two people, like love" | August 4 at last |

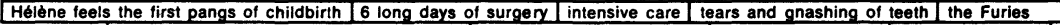
prowl about / men women and machines rush and shout 200 wills pierce the screen 6 long days on end 1 strange obstetrics terrifying demands from Pierre Morin, accoucheur-in-chief lafter so much suffering, she was born at dawn. Now, she must be carefully handled. recreation: in a religious community, or educ ational establishment, a time of rest and liberty accorded the monks, or pupils, for relaxation llooking ahead / redefining beginning |TO BE always in the future /perceiving $\mid 1$ receive, I absorb, I accomplish for a small, full, screen Imachines for perceiving machines for writing machines for storing memories 1 re-creation: action of mentally reconstructing September 5 in Chant d'Amours $/$ screening oh seeing the music \begin{tabular}{l|l|l|l|l|l|l|l|l}
\hline in the making & welling up like a spring & drinking & the eye listens & the ear sees & technique is forgotten & politeness & the song \\
\hline
\end{tabular} remains the work is finished $/$ not quite on his own the unknown viewer gives it life creation: action of making, organizing

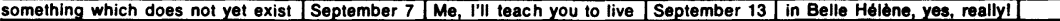
recording the credits $P$ an, with his balloons, awaits the unknown viewer $/$ Helene will really see the light of day one Sunday in December. At last. $\mid$ Now living every hall-second l let's go into the dark night in labyrinth Musical Offering in labyrinth Rite of Spring in labyrinth Garant's opera in labyrinth Hommage to Pierre Mercure in labyrinth in labyrinth in labyrinth \begin{tabular}{|l|lll|l|l|l}
\hline blood and light & Gabriel Charpentier, artistic adviser, music-television, Maison de Radio-Canada, Montréal. (c) \\
\hline
\end{tabular} 


\section{LE PARCOURS SINGULIER DE SERGE GARANT}

Une fois de plus, la mort est venue nous confondre et nous a laissés prostrés. Même si l'inéluctable de cette réalité "fait partie de la vie", nous ne pouvons nous y habituer ... et tout dans notre nature nous la fait craindre. Ceci, comme réflexe primordial, instinctif. Cependant, et c'est là également l'un des mystères de notre condition humaine, de ce désarroi même où nous sommes d'abord plongés en de telles circonstances surgit l'appétit de vivre, la foi, la passion et même, la joie. "Je pense que le grand avantage de l'itinéraire que j'ai fait, c'est qu'il repose strictement sur la passion", a écrit Serge Garant. Pour moi, c'est là son véritable testament : cette passion de vivre, d'agir, en un mot, de créer. Au-delà de toutes les polémiques, des jugements hâtifs ou des considérations d'ordre esthétique, voilà l'enseignement essentiel que nous a légué Serge Garant.

Quiconque a eu le privilège de travailler avec lui aura compris ce que le mot implication signifie : détermination, organisation et enthousiasme, se conjuguant de telle façon que nul ne pouvait demeurer imperméable à la portée de son action. Bien sûr, celle-ci fut sans doute intransigeante à certains moments, surtout au début de son combat pour l'expression contemporaine de la musique, mais cette intransigeance était pour lui la seule arme dont il pouvait se servir, à l'époque, afin de contrer l'immobilisme pernicieux d'un certain académisme qui refusait systématiquement de considérer de bonne foi toute expression musicale qui semblait renier les canons sacro-saints de la pensée traditionnelle. Il ne faut pas oublier qu'au début des années 50, dans notre milieu, la très grande majorité des musiciens ne connaissait même pas encore les principales oeuvres de Stravinsky ou de Bartók, pour ne nommer que ceux-là. Alors, que dire de l'Ecole de Vienne et, encore plus, de compositeurs tels que Messiaen et Boulez ? Il fallait donc un vigoureux coup de barre afin de favoriser une sérieuse prise de conscience de ce qui s'était passé et se passait au XXe siècle dans l'univers musical. Serge Garant a été le timonier de cette prise de conscience 
et la résistance qu'on lui a opposée était telle, dans plusieurs milieux, qu'il n'avait vraiment pas, tel que je l'ai écrit plus haut, le choix des armes : il fallait frapper, même à outrance parfois .... Qui, après tout, pourrait le lui reprocher ? L'honnêteté et la conviction de son geste étaient, de toute évidence, pour la bonne cause. Comme il l'a dit luimême : “... il fallait que je sois littéralement dévoré par cela pour oser le faire, car j'étais sans guide”.

J'estime que la fin de cette citation est révélatrice de l'attitude fondamentale de Serge Garant, en même temps qu'une explication de la nature de sa démarche : en effet, Serge "était sans guide", non seulement dans son activité de diffuseur de la musique contemporaine, mais également dans son propre travail de compositeur ; de ce point de vue, c'était une sorte d'autodidacte - malgré les leçons tirées des classes d'analyse de Messiaen - et c'est fort probablement à cause de cela qu'il a pu entreprendre et poursuivre, de la manière originale que l'on sait, son cheminement de compositeur et d'animateur. Il est venu à la musique sans préjugés, vierge en quelque sorte, et son évolution a été à rebours, ni plus ni moins, de celle généralement vécue par le musicien de formation traditionnelle. Cela a certainement influencé sa personnalité et lui a permis, en tout cas, d'accéder plus aisément et très rapidement à une expression créatrice typiquement actuelle. Il me semble évident que cette conjoncture particulière a joué un rôle déterminant dans le cheminement de l'homme : musicien polémiste-provocateur et, surtout, compositeur et pédagogue.

A plusieurs reprises, j'ai eu l'occasion de débattre ces questions avec lui lors de conversations intimes que nous avions de plus en plus fréquemment, soit à la faculté de musique de l'Université de Montréal, ou encore, dans l'ambiance chaleureuse de sa maison des Cantons de l'Est. Il n'était pas de ceux qui se laissent facilement aller aux confidences ; il ne recherchait pas non plus celles des autres. Cependant, ces dernières années, une grande amitié nous avait liés, qui permettait un échange beaucoup plus personnel et libre de toute contrainte. 
A la faveur de quelques-unes de ces recontres, il en est venu à considérer le parcours singulier de son évolution, y voyant notamment une cause plausible de la nature de ses opinions. Il commençait même à réaliser que ce qui avait été bénéfique pour lui ne l'était pas forcément pour les autres. Sa pudeur et sa modestie l'ont longtemps empêché de se voir tel qu'il était : un être exceptionnel et un musicien prodigieux pour qui les normes habituelles d'apprentissage ne s'appliquaient pas, pas plus que les résultats auxquels il était lui-même parvenu ne pouvaient s'appliquer aux autres. Il est important de souligner ce point : les Serge Garant ne courent pas les rues ... et nous devons pleinement réaliser que ce qu'il a été possible d'accomplir à cet esprit brillant l'a été précisément à cause de sa rare valeur ; s'imaginer le contraire serait faire preuve d'une dangereuse présomption.

L'oeuvre de Serge Garant ne peut vraiment se vérifier et s'apprécier que lorsqu'observée avec objectivité et lucidité, c'est-à-dire sans parti pris et sans fanatisme. Pour nous qui demeurons, que cette disparition ne soit point prétexte à amertume et à désenchantement ; ce serait là trahir la mémoire de celui que l'on a aimé et admiré. Ce serait aussi galvauder la portée du témoignage qu'il nous a laissé. Faisons plutôt nôtres cette détermination, cette force d'oeuvrer sans compromission, cette manière de toujours être à l'écoute de son intelligence et de son instinct profond. Car, finalement, Serge était fondamentalement un instinctif. A tel point que, craignant peut-être les excès de quelques débordements, il s'est forgé un métier et une rigueur de pensée qui garantissaient à ses yeux cet équilibre sans cesse recherché dans chacun de ses gestes de création.

André Prévost, O.C. le 8 décembre 1986 


\section{SERGE GARANT, A PIONEER}

Serge Garant was a pioneer among my generation of Canadian composers. Scores by him dating from 1954 show that he already discovered and was pacing Pierre Boulez ; and chronologically they placed him several years ahead of anyone else in this country in his understanding of what was then the newest music.

Music has changed a lot since then but Serge Garant's music changed very little except that it became more controlled, by which I do not mean more conservative, which it never became.

As a dynamic personality, he both raised and restructed new music undertaking in Quebec. No doubt, as is the case with all great human beings, his detractors will now emphasize the limitations of his vision. He did at least have a vision, a clear and unfogged vision, which has left its mark on Canadian music in some beautiful compositions, a style of musical interpretation and a never-to-be-forgotten legacy of witty aphorisms.

R. Murray Schafer December 11, 1986 


\section{SERGE GARANT, LE GRAND INCORRUPTIBLE}

J'ai accompagné Serge Garant lors de son dernier engagement comme chef d'orchestre en dehors du Canada, le 9 février 1985, pour un concert de musique canadienne (Garant, Tremblay, Vivier et moi-même) donné par le Nouvel Orchetre Philharmonique de Radio France. Je ne saurais trop souligner à quel point les musiciens étaient impressionnés par le travail de Serge. Il disaient : "Quel grand professionnel, quel merveilleux musicien ! Il doit avoir beaucoup d'engagements". Lorsque je leur ai répondu qu'il ne dirigeait que sept ou huit concerts par année et était obligé de faire de l'enseignement comme gagne-pain, ils sont restés incrédules.

Lors de ce voyage, nous avons beaucoup parlé de la situation et de l'évolution de la musique contemporaine. Serge disait : "Nous sommes au creux de la vague. La plupart des compositeurs improvisent au lieu de construire. Ils font des pastiches de vieille musique. Ils ne savent plus ce que c'est que la musique contemporaine. En tout cas, je trouve si déprimant le milieu actuel que je n'ai plus le courage d'écrire de la musique."

Serge était un homme d'action. Conditionné par ses années de travail comme arrangeur à Radio-Canada, il ne composait que si on lui demandait une oeuvre pour une date précise. Reconnu comme l'un des meilleurs compositeurs du Canada, il recevait pourtant très peu de commandes ces dernières années. Or, quand on regarde les médiocrités qui ont bénéficié de commandes importantes à la même époque, on a le droit de se poser bien des questions sur la vie musicale au Canada.

Homme d'une grande modestie, d'une certaine timidité, Serge était désavantagé dans un milieu où le plus effronté l'emporte. Il était d'une négligence totale envers sa propre musique. Ainsi, à la S.M.C.Q., il fallait l'obliger à mettre ses oeuvres au programme. Dans un monde pourri d'opportunistes, il restait le grand incorruptible, ne s'étant jamais servi de sa position pour faire avancer sa carrière. 
Puisque Serge s'est tellement battu pour défendre la musique d'autrui, il faut maintenant faire vivre sa musique. D'ailleurs, ayant dirigé son Quintette ainsi que Circuit III, pour 18 instruments, avec les élèves à l'Université McGill, j'ai constaté qu'on n'a pas besoin de virtuoses pour interpréter la plupart de ses oeuvres. Sa musique est accessible aux instrumentistes d'un bon niveau. Il n'y a jamais les maladresses de notation ou d'écriture instrumentale qui caractérisent beaucoup d'oeuvres contemporaines.

Bruce Mather le 29 novembre 1986 


\section{IF I COULD, I WOULD TELL SERGE ...}

The night before Serge Garant died, he was wandering in my dreams in a state of frustration because he had not finished his work. I spoke to Bruce Mather the next afternoon who confirmed that Serge was very ill and in hospital, and within 24 hours he succumbed. It was a shock and a great loss for everyone who knew him and his work. Personally, I was disturbed by this strong communication from my colleague on the eve of his death - I had not seen or talked to Serge for some months, I felt as if the dream was some kind of message.

If I could respond to that message, I would tell Serge how much I regret that he did not finish his work, work in the broadest sense, work that produced especially many inspired compositions and a rare conducting talent, work that revealed a profound commitment to contemporary music through teaching, broadcasting, and as long-time Artistic Director of the Société de musique contemporaine du Québec. I am saddened that we have lost you so prematurely Serge.

Norma Beecroft December 18, 1986 


\section{SERGE GARANT ： AN EXAMPLE THROUGHOUT CANADA}

Serge Garant was one of the most important musical figures of our generation, not only due to the music he composed, the performances he directed, the radio programmes he produced, or the composition and analysis classes he taught but because of the example he set. His highly principled artistic life served a catalytic function for musical activities across the entire country and even those Canadian musicians who did not know him personally or even experience his finely hewn music making, felt his presence.

News of his latest works, with their often original and carefully structured features spread far beyond the confines of his close circle of devotees and were often discussed in other parts of the country, without, more often than not, even having been heard. It was this same work which brought news of his conducting exploits, analytic and teaching prowess and of course the successes of the Société de musique contemporaine du Québec and pointed out, to many of us, the way to go.

I feel very fortunate, at having had the privilege of knowing Serge Garant and having experienced his music making first hand. Not only did he help my career through commissions, performances and general encouragement but once again by the example he set through an uncompromising attitude towards his own high artistic ideals. I am deeply saddened at his passing but when I think about Serge as he was, I am almost more pained by the fact that more people in our country were unable to know him better. 


\section{L'AUTHENTICITÉ DE SERGE GARANT}

Serge Garant fut un artiste authentique. Tout d'abord, à cause de son incroyable engagement, dès l'instant où il découvrit la musique du XXe siècle dans les années 40 . Un engagement qui rappelle l'illumination de Saint Paul et se manifesta autant dans ses compositions que dans sa carrière d'animateur, au sens le plus indicatif de ce terme.

A cet engagement s'ajouta l'importance notion de "service". Serge Garant fut au service de l'art : comme un soldat, il lutta au nom de la musique, il en fit la promotion. Bien sûr, d'autres compositeurs canadiens et québécois se mirent au service de la musique. Mais ce qui est terrible à mon avis dans le cas de Serge Garant, c'est qu'il joua un peu le rôle de Cassandre : il disait la vérité, mais personne ne le croyait. La meilleure preuve en est que la Société Royale du Canada, en avril dernier, l'honora à titre de musicologue et non de compositeur. Fait qu'il ne manqua pas de souligner.

Serge Garant avait une connaissance profonde de la musique de son époque. En sa qualité de directeur artistique de la Société de musique contemporaine du Québec, il établit des standards très élevés. Avec le résultat que, pour un compositeur comme moi, par exemple, savoir qu'il existait un organisme comme la S.M.C.Q., et que celui-ci était dirigé par Serge, représentait une expérience de libération : l'héritage qu'il laisse est que la S.M.C.Q. constitue peut-être le seul lieu au Canada, sinon en Amérique du Nord, où l'on sait que notre musique recevra une interprétation de tout premier ordre. On est libéré de la médiocrité, de la confusion, de l'obscurité, des boulets qui empêchent souvent d'être un compositeur. Bref, libération de l'absence de bonne volonté.

Garant fut aussi un leader. Il était capable de convaincre ceux qui travaillaient avec lui que la nouvelle musique était quelque chose de bien réel et de bien vivant. Pour toute une génération de musiciens, il fut le meilleur des professeurs : chose rare, il était à la fois un guide et un complice. 
On oublie souvent de mentionner le mot "art" lorsqu'on parle de Serge Garant ; on parle seulement de "musique" . Pourtant, ses compositions relèvent du domaine de l'art. Ce qui représente un défi beaucoup plus considérable, ne serait-ce que parce qu'il est plus dangereux.

John Rea

le 15 décembre 1986 


\section{SERGE GARANT, INCROYABLE TRAVAILLEUR DE LA MUSIQUE}

Lorsque, en 1966, un surcroît de travail administratif à la faculté de musique de l'Université de Montréal m'a forcé à me retirer temporairement de l'enseignement de la composition, c'est à Serge Garant que j'ai voulu confier ma classe. Et c'est à cette même époque que j'ai commencé à travailler avec lui à la Société de musique contemporaine du Québec. J'ai donc pu constater de très près non seulement ses qualités de compositeur, de professeur, d'animateur et de chef d'orchestre, mais aussi son incroyable capacité de travail et son dévouement.

Plus particulièrement, je voudrais souligner que c'est un des plus grands analystes de la musique du XXe siècle qui nous a quittés. Sa perte est très cruelle pour la Société de musique contemporaine du Québec, pour la faculté de musique de l'Université de Montréal et pour la musique du Québec, du Canada et du monde. 


\section{SERGE GARANT, PENSEUR DE LA MUSIQUE DU XXe SIÈCLE}

Je connus Serge Garant en 1970, alors que je suivais ses cours d'analyse à la faculté de musique de l'Université de Montréal. Son enseignement était admirable. L'accent était mis sur les oeuvres des Viennois, mais y prenaient place aussi d'autres contemporains : Varèse, Messiaen, Stockhausen, etc. Pour moi, qui avais à ce moment-là une formation traditionnelle, ce fut une expérience très enrichissante. Garant avait une formidable capacité pour décortiquer les musiques structurées, notamment celles de Webern. Il communiquait son enthousiasme à toute la classe et la plupart des élèves étaient conscients d'assister à des cours d'une grande importance.

D'autre part, au même moment, je poursuivais une démarche en composition très éloignée des "préceptes" de Garant. Peu à peu, j'ai adopté une méthode de travail plus rationnelle et systématique, et ce n'est que bien des années plus tard que je me suis rendu compte qu'il s'agissait là d'une influence de Garant, influence qui s'était manifestée presque comme un mécanisme à retardement.

Tout le monde reconnait l'importance capitale de Serge Garant comme compositeur et chef d'orchestre. Je pense qu'il ne faudrait pas oublier non plus le Serge Garant penseur : celui qui s'est penché sur les problèmes et les processus de la création musicale et a su faire partager aux autres ses interrogations et ses découvertes. 


\section{L'ACTION IRREMPLAÇABLE DE SERGE GARANT}

Il n'est pas nécessaire d'énumérer les multiples implications de Serge Garant dans le milieu musical ; chacun a eu l'occasion de connaître ce musicien à travers l'une ou l'autre de ses nombreuses activités : compositeur, chef d'orchestre, professeur, animateur radiophonique, etc.. Le rayonnement de Serge Garant a influencé le milieu musical québécois durant de longues années. Directeur artistique de la Société de musique contemporaine du Québec, Serge Garant se trouvait ainsi en première position pour choisir, décider et présenter au public des oeuvres d'avant-garde. Quant à l'émission "Musique de notre siècle", elle lui permettait de faire la critique des nouvelles partitions. Cette position de force n'était pas sans imposer à son "leader" une grande responsabilité : était-ce celle d'éduquer ou d'informer le public ? Il est évidemment difficile de faire l'un sans l'autre. En effet, le public doit être mis au courant des nouvelles techniques, de ce qui se fait ailleurs et de toute innovation susceptible d'animer la vie culturelle québécoise. Mais cette information n'est pas sans comprendre en même temps le risque d'influencer. Et à ce niveau, les goûts de Serge Garant ont profondément marqué non seulement ses étudiants, mais les mélomanes avertis qui, peut-être, ont parfois pris parti pour une certaine esthétique davantage pour suivre une mode que par goût personnel. Ce n'est pas un reproche que j'adresse à Serge Garant. Je crois au contraire que le musicien tout comme l'homme étaient parfaitement honnêtes. Absolument convaincu du bien-fondé de ses pensées qui se voulaient avant tout structurelles, Serge Garant a persuadé l'autre de ce qu'il tenait lui-même pour absolu. Cette rigueur artistique qu'il s'était imposée a pu être perçue comme absence de sensibilité. Pour avoir étudié avec lui durant deux ans, je me suis rendu compte que derrière une image qui, de prime abord pouvait paraître austère, se cachait en fait une grande sensibilité, disponibilité et générosité. Ce n'est que ces dernières années que Serge Garant a élargi les règles qu'il s'était lui-même imposées. Je pense à Plages, oeuvre pour orchestre d'un lyrisme inhabituel chez lui. 
Il nous a quittés trop tôt : trop tôt pour lui qui n'a pas eu le temps d'exploiter à fond ce retour à une musique plus linéaire, et trop tôt pour nous puisqu'il ne pourra pas nous communiquer à nouveau ses découvertes. Son action dans le milieu québécois reste irremplaçable et sa musique témoigne du cheminement d'un compositeur parti d'une esthétique rigoureuse vers une plus grande liberté.

Anne Lauber

le 3 décembre 1986 


\section{LA MAGNIFIQUE OBSTINATION DE SERGE GARANT}

Il n'est pas facile, certes, de se conditionner pour dire adieu à un ami, un compagnon d'armes - puisque nos carrières furent parallèles mais surtout de perdre un homme digne, généreux jusqu'à être prodigue et toujours, en tant que musicien, à l'écoute de tous.

Prophète d'une musique nouvelle dans cette Amérique française, Serge Garant se fait le porte-parole de ses compagnons d'alors pour établir des bases et convaincre un monde musical bien provincial et nostalgique de la nécessité de l'ascèse de l'écriture et de l'action menée en faveur de ce que l'on jugeait important, essentiel dans la musique du XXe siècle. Obstiné à se découvrir lui-même, Garant fait la preuve par neuf, démontrée par une série d'oeuvres d'une rare modernité, dans cet aéorogare de la musique canadienne "où tous les styles se donnent rendez-vous, en attendant le prochain départ" (Garant : 1959).

Dans un article intitulé "Musicien structuraliste et musicien lyrique" (Morel : 1978), j'affirmais que la lecture d'une oeuvre de Serge Garant était et demeurera pour moi aussi enrichissante que la lecture de partitions de maîtres, reconnus comme tels, de la deuxième moitié du XXe siècle. Je crois en outre juger parfois sévèrement mes collègues canadiens pour leur manque de vision, parfois pour leur attitude "showbiz". Mais à l'encontre de la majorité d'entre eux, Serge Garant ne m'a jamais déçu. Son attitude vis-à-vis de la création musicale fut toujours marquée d'une écriture empreinte de rigueur, sans égard pour les diverses modes décidées ailleurs et sans grand lendemain. Vous découvrez maintenant que Serge Garant fut et demeure un créateur de premier ordre dans ce jeune pays. Eh! bien, comme à l'habitude dans 
tous ces petits mondes musicaux marginaux, dans une civilisation qui s'écroule par sa médiocrité, cela ne surprend guère. Car c'est le côté affligeant du quotidien d'un compositeur du XXe siècle qu'on le découvre après sa mort, afin d'avoir un certain ... recul. Mieux vaut trop tard que jamais.

Salut, Serge Garant.

François Morel

le 11 décembre 1986

\section{RÉFÉRENCES}

GARANT, $S$.

1959 : "Dire une musique d'ici", Cahier pour un paysage à inventer I, 53-55.

MOREL, F.

1978 : "Musicien structuraliste et musicien lyrique", Variations II, 3. 


\section{SERGE GARANT, ÊTRE DE RIGUEUR ET D'ÉMOTION}

Très cher ami et compagnon de route ! Faut-il parler de toi au passé, toi le passionné du présent, là où se manifeste la musique en ce temps où nous restons, bouleversés?

C'est chez moi que nous nous sommes rencontrés pour la première fois avec François Morel et Roger Matton qui tenait à nous présenter ce musicien phénoménal de Sherbrooke. Nous étions alors dans la jeune vingtaine et venions de découvrir Varèse. Arrivant de Paris, tu apportais dans ta serviette des partitions de Messiaen, de Webern, alors à peu près inconnus ici, ainsi que du jeune Boulez. Tu nous parlais aussi de Stockhausen. Ce fut une journée exaltante. Lisant, jouant, improvisant au piano, en une sorte de passion juvénile où, questions, doutes, critiques, adhésion entremêlés, soufflait l'enthousiasme. De plus, nous avions l'impression de nous mettre à l'heure du monde. Basé sur cette ferveur partagée, tel fut le point de départ de notre amitié, une amitié assez solide pour se permettre toutes les divergences. Nos chemins différaient, mais à chaque croisée, un accord profond nous réunissait tacitement, sans que nous ayons besoin de l'expliciter.

Au nom de tous tes amis, collaborateurs et collaboratrices, proches ou lointains, je tiens à te rendre hommage pour ce don d'amitié.

Chef d'orchestre, membre fondateur et directeur artistique de la Société de musique contemporaine depuis vingt ans, pédagogue, professeur à l'Université de Montréal, commentateur à la radio, toutes ces activités dérivaient du fait que tu étais avant tout compositeur. Et c'est à celui-ci qu'il faut surtout rendre hommage pour la très haute exigence et la rigueur que tu avais envers toi-même, soucieux de netteté et d'exactitude. Etre d'émotion, tu aimais dire "l'émotion, bien sûr que ça existe, mais j'ai horreur d'en parler". Le lyrisme de pudeur qui t'est propre n'en est que plus éloquent. 
Quant au chef d'orchestre, tous les musiciens qui ont travaillé sous ta direction ont loué ta technique d'une rare clarté. Mais le contact était aussi d'un autre ordre : les musiciens t'aimaient.

Pour les compositeurs, travailler avec un tel chef devenait une collaboration de tous les instants. Serge Garant est sûrement le chef de ce pays qui a monté le plus d'oeuvres nouvelles d'ici et de l'étranger. Associés à ceux d'ici, les témoignages de Pierre Boulez, Gilbert Amy, Claude Ballif, Xénakis, Claude Helffer, François-Bernard Mâche, Marius Constant, Mariano Etkin, Norma Beecroft, John Beckwith, Robert Aitken, et de plusieurs autres qui nous arrivent, expriment leur consternation et leur reconnaissance.

Je tiens aussi à mentionner, en présence de ses frères et soeurs, l'importance qu'avait sa famille pour lui, surtout ces dernières années. N'est-ce pas vous tous qui, avec ses intimes les plus dévoués, Louise Hirbour, Jean-Paul Néron, l'avez assisté dans cette mort très douce ? Serge m'avait quelquefois parlé aussi de son père, qui fut membre de l'Harmonie de Sherbrooke, et de sa mère qui était, paraît-il, une excellente musicienne.

Serge Garant s'était vu décerner plusieurs honneurs et décorations, dont le Prix Calixa-Lavallée de la Société Saint-Jean-Baptiste et le Prix Jules-Léger, pour ne citer que ceux-là. Il venait également d'être reçu membre de la Société Royale du Canada. Si ces événements ont un sens certain, ne serait-ce que celui de la reconnaissance, ils restent malgré tout extérieurs aux véritables événements, qui se passent dans le secret le plus intime et dans la solitude : une idée qui naît, l'engagement de la volonté, ce dont on ne parle jamais mais qui mérite d'être mentionné avec le plus grand respect.

Pendant sa longue maladie, Serge Garant a reçu de nombreuses lettres d'amis, de collègues, et surtout d'élèves anciens ou récents. Il a été surpris de découvrir qu'on l'aimait à ce point et cela l'a aidé, a-t-il dit, à supporter l'épreuve de ces derniers mois. Parmi ceux qui ont compati à sa maladie, un de nos collaborateurs nous a également quittés il y a 
trois mois : je voudrais en ce jour associer Daniel Sarijian à ce tissu d'amour.

"C'est en Adam, dit saint Paul, que meurent tous les hommes ; c'est dans le Christ que tous revivront, mais chacun en son rang : en premier le Christ ; et ensuite, ceux qui seront au Christ lorsqu'il reviendra. Alors, tout sera achevé, quand le Christ remettra son pouvoir royal à Dieu le Père, après avoir détruit toutes les puissances du mal. C'est lui en effet qui doit régner jusqu'au jour où il aura mis sous ses pieds tous ses ennemis. Et le dernier ennemi qu'il détruira, c'est la mort, car il a tout mis sous ses pieds." (I, Cor, 15, 20-27a)

Serge, ayant toi-même désiré cette cérémonie, tu as voulu qu'y soit incorporée ton oeuvre ...chant d'amours. Ce chant d'amour, "soif d'essentiel" comme l'a mentionné le Père Louis Cyr, n'est-il pas ton testament?

Gilles Tremblay le 8 novembre 1986 


\section{SERGE GARANT ET LES AUTRES}

Je reconnais en Serge Garant le compagnon des premières heures, qui s'est voué avec une totale générosité à la cause de la musique contemporaine. Il ne s'est pas contenté de décider et de choisir pour lui, mais, ayant pris cette décision quant à lui-même, basée sur une réflexion et sur une fraternité, il a tenu à faire partager ses points de vue qu'il considérait, à juste titre, comme les mieux adaptés à notre temps, comme les plus susceptibles de donner des territoires nouveaux à la musique, territoires dont elle a sans cesse besoin. Cela implique les multiples besognes du quotidien, le dévouement qui supprime l'égoïsme quelquefois tellement désirable et désiré, cela nécessite des options et des prises de position qui peuvent, et même doivent, heurter un conformisme n'ayant que trop tendance à se satisfaire de ce qui est. Rien à regretter ! C'est de cela qu'est faite la vie, inextricablement : soi et les autres, et, dominant tout cela, un idéal auquel il n'a pas sacrifié, mais bien consacré son activité, ses dons et sa passion. Qu'il en soit remercié même par ceux qui n'ont pas participé directement à son activité, qui l'ont connue par l'écho qu'elle provoquait. Savoir cette solidarité, au loin, n'était peut-être qu'une mince récompense dans l'immédiat, mais elle l'inscrit, maintenant que sa vie est achevée, dans cet irrésistible mouvement que la curiosité et la passion des hommes les plus volontairement illuminés ont toujours suscité.

Pierre Boulez

le 2 novembre 1986 